\title{
Existence Theorem for a Nonlinear Functional Integral Equation and an Initial Value Problem of Fractional Order in $L_{1}\left(R_{+}\right)$
}

\author{
Ibrahim Abouelfarag Ibrahim ${ }^{1,2}$, Tarek S. Amer ${ }^{1,3}$, Yasser M. Aboessa ${ }^{1,4}$ \\ ${ }^{1}$ Mathematics Department, Faculty of Science and Education, Taif University, Al-Khurmah Branch, Taif, KSA \\ ${ }^{2}$ Mathematics Department, Faculty of Science, Suez Canal University, Ismailia, Egypt \\ ${ }^{3}$ Mathematics Department, Faculty of Science, Tanta University, Tanta, Egypt \\ ${ }^{4}$ Mathematics Department, Faculty of Science, Al-Azhar University, Cairo, Egypt \\ Email: iabouelfarag@hotmail.com, tarekamer30@hotmail.com,dd_yasser@yahoo.com
}

Received November 21, 2012; revised December 21, 2012; accepted December 30, 2012

\begin{abstract}
The aim of this paper is to study the existence of integrable solutions of a nonlinear functional integral equation in the space of Lebesgue integrable functions on unbounded interval, $L_{1}\left(R_{+}\right)$. As an application we deduce the existence of solution of an initial value problem of fractional order that be studied only on a bounded interval. The main tools used are Schauder fixed point theorem, measure of weak noncompactness, superposition operator and fractional calculus.
\end{abstract}

Keywords: Nonlinear Functional Integral Equation; Volterra Operator; Measure of Weak Noncompactness; Fractional Calculus; Schauder Fixed Point Theorem

\section{Introduction}

The class of functional integral equations of various types plays very important role in numerous mathematical research areas. An interesting feature of functional integral equations is its role in the study of many problems of functional differential Equations [1-4].

In this work we study the solvability of the following initial value problem

$$
\begin{aligned}
& \frac{\mathrm{d} y(t)}{\mathrm{d} t}=\int_{0}^{t} k_{1}(t, s) f\left(s, D^{\beta} y(s)\right) \mathrm{d} s, \\
& t \in R_{+}, \beta \in(0,1], y(0)=y_{0} .
\end{aligned}
$$

where $D^{\beta} y$ denotes the fractional derivative of order $\beta$ of $y$ with $\beta \in(0,1]$. Such initial value problem of arbitrary order (1) was investigated in [5-7]. To achieve this goal, let us consider the integral equation

$$
\begin{aligned}
& x(t)=\int_{0}^{t} k_{1}(t, s) f\left(s, \int_{0}^{s} k_{2}(s, \theta) x(\phi(\theta)) \mathrm{d} \theta\right) \mathrm{d} s, \\
& t \in R_{+}
\end{aligned}
$$

which is different from that studied in [2].

Section 2 contains some basic results. Our main result will be given in Section 3. Solvability of the considered initial value problem will be discussed in Section 4 .

\section{Basic Concepts}

This section is devoted to recall some notations and known results that will be needed in the sequel.

If $A$ is a Lebesgue measurable subset of the set of real numbers $R$ then we use the symbol meas. $(A)$ to denote the Lebesgue measure of $A$. Let $L_{1}(A)$ be the space of all real functions defined and Lebesgue measurable on the set $A$. If $x \in L_{1}(A)$ then the norm of $x$ is defined as:

$$
\|x\|=\|x\|_{L_{1}(A)}=\int_{A}|x(t)| \mathrm{d} t .
$$

when $A=R_{+}=[0, \infty)$, we will write $L_{1}$ instead of $L_{1}\left(R_{+}\right)$.

\subsection{The Superposition Operator}

An important operator called the superposition operator can be investigated in the theories of differential integral and functional equations [4,8-10]. It can be defined as follows:

Definition 1. Assume that $f: I \times R \rightarrow R$ satisfies Carathéodory conditions, that is it is measurable in $t$ for any $x$ and continuous in $x$ for almost all $t$ where $t \in I, x \in R$. Then for every measurable function $x$ on the interval $I$ we assign the function:

$$
(F x)(t)=f(t, x(t)), t \in I .
$$


The operator $F$ defined in this way is called the superposition operator generated by the function $f$.

Carathéodory [11] gave the first contribution to the theory of the superposition operator and proved its measurability according to the measurability of $f$.

We state the following result giving the necessary and sufficient condition so that the superposition operator $F$ generated by $f$ will map continuously $L_{1}$ into itself [12].

Theorem 2. Let $f$ satisfy the conditions in Definition 1. The superposition operator $F$ generated by the function $f$ maps continuously the space $L_{1}$ into itself if and only if:

$$
|f(t, x)| \leq a(t)+b|x|,
$$

for all $t \in I$ and $x \in R$, where $a$ is a function that belongs to $L_{1}$ and $b$ is a nonnegative constant.

It is known that a real valued continuous function is measurable and that the converse is not necessarily true. However, for the converse we have the following results due to Dragoni [13].

Theorem 3. Let $I$ be a bounded interval and $f: I \times R \rightarrow R$ be a function satisfying Caratheodory conditions. Then for each $\varepsilon>0$ there exists a closed subset $D_{\varepsilon}$ of the interval $I$ such that meas. $\left(I / D_{\varepsilon}\right)<\varepsilon$ and $\left.f\right|_{D_{\varepsilon} \times R}$ is continuous.

\subsection{Volterra Integral Operator}

We proceed by recalling some basic facts concerning the linear Volterra integral operator in the Lebesgue space $L_{1}$. Suppose $k: \Delta \rightarrow R$ is a given function which is measurable with respect to both variables where

$$
\Delta=\{(t, s): 0 \leq s \leq t<\infty\} .
$$

For an arbitrary function $x \in L_{1}$ define Volterra integral operator as follows:

$$
(K x)(t)=\int_{0}^{t} k(t, s) x(s) \mathrm{d} s, t \in R_{+} .
$$

It is well known that if $K: L_{1} \rightarrow L_{1}$ then it is continuous $[4,9]$.

In general, it is rather difficult to find necessary and sufficient conditions for the function $k(t, s)$ guaranteeing that the integral operator $K$ transforms the space $L_{1}$ into itself. Some special cases of this problem were discussed in $[4,14]$. In this direction we state the next result [15]:

Theorem 4. Let $k$ be measurable on $\Delta$ and such that

$$
\underset{s \geq 0}{\operatorname{ess} \sup } \int_{s}^{\infty}|k(t, s)| \mathrm{d} s<\infty .
$$

Then the Volterra integral operator $K$ generated by $k$ maps (continuously) the space $L_{1}=L_{1}\left(R_{+}\right)$into itself and the norm $\|K\|$ of this operator is majorized by the number

$$
\underset{s \geq 0}{\operatorname{ess} \sup } \int_{s}^{\infty}|k(t, s)| \mathrm{d} s .
$$

Observe that if $D$ is a nonempty and measurable subset of $R_{+}$then we can also consider the linear Volterra integral operator associated with the Lebesgue space $L_{1}(D)$.

Namely,

If $x \in L_{1}(D)$ where $D$ is a nonempty and measurable subset of $R_{+}$then we extend $x$ to the whole half axis $R_{+}$by putting $x(t)=0$ for $t \in R_{+} / D$. Then we can treat $K$ in the usual way. When the operator $K$ transforms $L_{1}(D)$ into itself its norm will be denoted by $\|K\|_{D}$.

\subsection{Measures of Weak Noncompactness}

Let us assume that $E$ is an infinite dimensional Banach space with the norm $\|\cdot\|$ and the zero element $\theta$. Denote by $m_{E}$ the family of all nonempty and bounded subsets of $E$ and by $n_{E}^{W}$ its subfamily consisting of all relatively weakly compact sets. The symbol $\bar{X}^{W}$ stands for the weak closure of a set $X$ and the symbol ConvX will denote the convex closed hull (with respect to the norm topology) of a set $X$. We denote by $B(x, r)$ the ball centered at $x$ and of radius $r$. We write $B_{r}$ instead of $B(\theta, r)$. In what follows we accept the following definition [16]

Definition 5. A function $\mu: m_{E} \rightarrow R_{+}$is said to be a measure of weak noncompactness if it satisfies the following conditions: The Family

1) The family $\operatorname{ker} \mu=\left\{X \in m_{E}: \mu(X)=0\right\}$ is nonempty and is nonempty and ker $\mu \subset \bar{n}_{E}^{W}$.

2) $X \subset Y \Rightarrow \mu(X) \leq \mu(Y)$.

3) $\mu(\operatorname{Conv} X)=\mu(X)$.

4) $\mu(\lambda X+(1-\lambda) Y) \leq \lambda \mu(X)+(1-\lambda) \mu(Y)$, for $\lambda \in[0,1]$.

5) If $X_{n} \in m_{E}, X_{n}=\bar{X}_{n}^{W}$ and $X_{n+1} \subset X_{n}$ for $n=1,2, \cdots$

And if $\lim _{n \rightarrow \infty} \mu\left(X_{n}\right)=0$ then the intersection is nonempty $X_{\infty}=\bigcap_{n=1}^{\infty} X_{n}$

The family $\operatorname{ker} \mu$ is said to be the kernel of the measure of weak noncompactness $\mu$. Let us observe that the intersection set $X_{\infty}$ from 5) belongs to ker $\mu$. Indeed, since $\mu\left(X_{\infty}\right) \leq \mu\left(X_{n}\right)$ for every $n$ then we have that $\mu\left(X_{\infty}\right)=0$.

We can construct a useful measure of weak noncompactness in the space $L_{1}$ that based on the following criterion for weak noncompactness due to Dieudonné 
$[17,18]$.

Theorem 6. A bounded set $X$ is relatively weakly compact in $L_{1}$ if and only if the following two conditions are satisfied:

a) for any $\varepsilon>0$ there exists $\delta>0$ such that if meas. $(D) \leq \delta$ Then $\int_{D}|x(t)| \mathrm{d} t \leq \varepsilon$ for all, $x \in X$.

b) for any $\varepsilon>0$ there is $T>0$ such that $\int_{T}^{\infty}|x(t)| \mathrm{d} t \leq \varepsilon$ for any,$x \in X$.

Now, for a nonempty and bounded subset $X$ of the space $L_{1}$ let us define:

$$
\mu(X)=c(X)+d(X),
$$

where

$$
c(X)=\lim _{\varepsilon \rightarrow 0}\left\{\sup _{x \in X}\left\{\sup \left\{\iint_{D}|x(t)| \mathrm{d} t: D \subset R_{+} \text {, meas } D \leq \varepsilon\right\}\right\}\right\},
$$

and

$$
d(X)=\lim _{T \rightarrow \infty}\left\{\sup \left\{\int_{T}^{\infty}|x(t)| \mathrm{d} t: x \in X\right\}\right\} .
$$

It can be shown [17] that the function $\mu$ is a measure of weak noncompactness in the space $L_{1}$ such that $\beta(X) \leq \mu(X) \leq 2 \mu(X)$, for any $X \in m_{L_{1}}$, where $\beta$ denotes the De Blasi measure of weak noncompactness in $L_{1}$. Moreover, $\mu\left(B_{r}\right)=2 r$.

In our approach we will need the following fixed point theorem due to Schauder.

Theorem 7. Let $C$ be a nonempty, convex, closed, and bounded subset of a Banach space $E$. Let $H: C \rightarrow C$ be a completely continuous mapping. Then $H$ has at least one fixed point in $C$.

\subsection{Fractional Calculus}

The definitions of both differential operator and the integral operator of fractional order are stated as follows $[19,20]$.

Definition 8. Let $f \in L_{1}, \alpha \in R_{+}$. The RiemmanLiouville (R-L) fractional integral of the function $f(t)$ of order $\alpha$ is defined as

$$
\begin{aligned}
& I_{a}^{\alpha} f(t)=\int_{a}^{t} \frac{(t-s)^{\alpha-1}}{\Gamma(\alpha)} f(s) \mathrm{d} s, \\
& \alpha>0, a \leq t \leq b .
\end{aligned}
$$

Definition 9. Let $g(t)$ be an absolutely continuous function on $[a, b]$. Then the fractional derivative of order $\alpha \in(0,1]$ of $g(t)$ is defined as

$$
\begin{aligned}
& D_{a}^{\alpha} g(t)=I_{a}^{1-\alpha} D g(t), \\
& D=(\mathrm{d} / \mathrm{d} t) .
\end{aligned}
$$

We state here some results concerning the above mentioned operators:

1) Let $f, D f \in L_{1}$ and $\alpha, \beta \in(0,1]$, then

i) then $I_{a}^{\alpha} I_{a}^{\beta} f(t)=I_{a}^{\alpha+\beta} f(t)$.

ii) $D I_{a}^{\alpha} f(t)=I_{a}^{\alpha} D f(t)$, when $f(a)=0$.

2) The operator $I_{a}^{\alpha}$ maps $L_{1}$ into itself continuously.

\section{Existence Theorem}

Consider the integral Equation (2) and let $H$ denotes the operator determined by the right hand side of this equation, i.e.,

$$
(H x)(t)=\int_{0}^{t} k_{1}(t, s) f\left(s, \int_{0}^{s} k_{2}(s, \theta) x(\varphi(\theta)) \mathrm{d} \theta\right) \mathrm{d} s,
$$

where $t \in R_{+}$In fact the operator $H$ can be written as the product $H=K_{1} F_{1} K_{2} F_{2}$ of the linear Volterra operator

$$
\left(K_{i} x\right)(t)=\int_{0}^{t} k_{i}(t, s) x(s) \mathrm{d} s, i=1,2 .
$$

and the superposition operator

$$
(F x)(t)=f(t, x(t)), t \in R_{+} .
$$

Therefore Equation (4) can be written as:

$$
x=H x=K_{1} F_{1} K_{2} F_{2} x(\varphi) .
$$

To establish our main result concerning existence of an integrable solution of Equation (2) we impose suitable conditions on the functions involved in that equation. Namely we assume

1) The functions $f: R_{+} \times R \rightarrow R$ satisfy the Caratheodory conditions and there exist functions $a \in L_{1}$ and constants $b>0$ such that

$$
|f(t, x)| \leq a(t)+b|x|
$$

holds for all $(t, x) \in R^{+} \times R$.

2) The functions $k_{i}: R_{+} \times R_{+} \rightarrow R$ satisfy the Caratheodory conditions and the linear Volterra operators $K_{1}, K_{2}$ associated with $k_{1}, k_{2}$ map $L_{1}$ into itself.

3) $\varphi: R_{+} \rightarrow R_{+}$is increasing, absolutely continuous and there exists a constant $M>0$ such that $\varphi^{\prime}(t) \geq M$ a.e. on $R_{+}$.

4) $b\left\|K_{1}\right\|\left\|K_{2}\right\| M^{-1}<1$.

Now we can state our main result in the next theorem.

Theorem 10. Under the above assumptions the Equation (2) has at least one solution $x \in L_{1}$.

Proof. Since $H$ is a nonlinear operator defined by Equation (5), then based on assumptions i) and ii) if $x \in L_{1}$, then $H x \in L_{1}$. Moreover, from Equation (5), and noting that $K_{1}, K_{2}$ according to our assumptions are 
indeed bounded, we have

$$
\begin{aligned}
\|H x\| & =\left\|K_{1} F_{1} K_{2} F_{2} x(\varphi)\right\| \leq\left\|K_{1}\right\|\left\|F_{1} K_{2} F_{2} x(\varphi)\right\| \\
& \leq\left\|K_{1}\right\| \int_{0}^{\infty}\left|f\left(s, \int_{0}^{s} k_{2}(s, \theta) x(\varphi(\theta)) \mathrm{d} \theta\right)\right| \mathrm{d} s \\
& \leq\left\|K_{1}\right\| \int_{0}^{\infty}\left\{a(s)+b \int_{0}^{s} k_{2}(s, \theta) x(\varphi(\theta)) \mathrm{d} \theta \mid\right\} \mathrm{d} s \\
& \leq\left\|K_{1}\right\|\|a\|+b\left\|K_{1}\right\|\left\|K_{2} x(\varphi)\right\| \\
& \leq\left\|K_{1}\right\|\|a\|+b\left\|K_{1}\right\|\left\|K_{2}\right\| \int_{0}^{\infty}|x(\varphi(\theta))| \mathrm{d} \theta \\
& \leq\left\|K_{1}\right\|\|a\|+b\left\|K_{1}\right\|\left\|K_{2}\right\| \int_{0}^{\infty}|x(\varphi(\theta))| \frac{\varphi^{\prime}(\theta)}{M} \mathrm{~d} \theta \\
& \leq\left\|K_{1}\right\|\|a\|+b\left\|K_{1}\right\|\left\|K_{2}\right\| M^{-1} \int_{0}^{\infty}|x(u)| \mathrm{d} u \\
& \leq\left\|K_{1}\right\|\|a\|+b\left\|K_{1}\right\|\left\|K_{2}\right\| M^{-1}\|x\| .
\end{aligned}
$$

The above estimate shows that the operator $H$ maps $B_{r}$ into itself, where

$$
r=\left(\left\|K_{1}\right\|\|a\|(1-b)\left\|K_{1}\right\|\left\|K_{2}\right\| M^{-1}\right)^{-1} .
$$

Moreover, according to Theorem 2, we deduce that the operator $H$ is continuous on the space $L_{1}$.

Next, to prove that $H$ is a contraction, let $X$ be a nonempty subset of $B_{r}$. Fix $\varepsilon>0$ and take a measurable subset $D \subset R_{+}$such that meas. $D \leq \varepsilon$. Then for any $x \in X$, we get

$$
\begin{aligned}
& \int_{D}|(H x)(t)| \mathrm{d} t \\
& =\int_{D}\left|\left(K_{1} F K_{2} x(\varphi)\right)(t)\right| \mathrm{d} t \leq\left\|K_{1}\right\|_{D} \|\left. F_{1} K_{2} x(\varphi)\right|_{L_{1}(D)} \\
& \leq\left\|K_{1}\right\|_{D} \int_{D}\left|f\left(s, \int_{0}^{s} k_{2}(s, \theta) x(\varphi(\theta)) \mathrm{d} \theta\right)\right| \mathrm{d} s, \\
& \leq\left\|K_{1}\right\|_{D} \int_{D}\left|a(s)+b \int_{0}^{s} k_{2}(s, \theta) x(\varphi(\theta)) \mathrm{d} \theta\right| \mathrm{d} s \\
& \leq\left\|K_{1}\right\|_{D} \int_{D}|a(t)| \mathrm{d} t+b\left\|K_{1}\right\|_{D} \int\left|\left(K_{D} x(\varphi)\right)(t)\right| \mathrm{d} t \\
& \leq\left\|K_{1}\right\|_{D} \int_{D}|a(t)| \mathrm{d} t+b\left\|K_{1}\right\|_{D}\left\|K_{2} x(\varphi)\right\|_{L_{1}(D)} \\
& \leq\left\|K_{1}\right\|_{D} \int_{D}|a(t)| \mathrm{d} t+b\left\|K_{1}\right\|_{D}\left\|K_{2}\right\|_{D}\|x(\varphi)\|_{L_{1}(D)} \\
& \leq\left\|K_{1}\right\|_{D} \int_{D}|a(t)| \mathrm{d} t+\frac{b\left\|K_{1}\right\|_{D}\left\|K_{2}\right\|_{D}}{M} \int_{\varphi(D)}|x(u)| \mathrm{d} u
\end{aligned}
$$

where the symbol $\|\cdot\|_{D}$ denotes the operator norm acting from the space $L_{1}(D)$ into itself. Also in the above calculation we used the fact that $a(t) \geq 0$ for $t \in R_{+}$. From the absolute continuity of the function $\varphi$ and the obvious equality

$$
\lim _{\varepsilon \rightarrow 0}\left\{\sup \left\{\int_{D} a(t) \mathrm{d} t: D \subset R_{+}, \text {meas. } D \leq \varepsilon\right\}\right\}=0 .
$$

and using Theorem 6 we obtain

$$
c(H X) \leq\left(\frac{b\left\|K_{1}\right\|_{D}\left\|K_{2}\right\|_{D}}{M}\right) c(X) .
$$

Furthermore, fixing $T>0$ we can deduce that

$$
\begin{aligned}
& \int_{T}^{\infty}|(H x)(t)| \mathrm{d} t \\
& =\left\|K_{1} F K_{2} x(\varphi)\right\|_{L_{1}[T, \infty)}=\int_{T}^{\infty}\left|\left(K_{1} F K_{2} x(\varphi)\right)(t)\right| \mathrm{d} t \\
& \leq K_{1 T} \int_{T}^{\infty}|a(t)| \mathrm{d}+\frac{b K_{1 T} K_{2 T}}{M} \int_{\varphi(T)}^{\infty}|x(u)| \mathrm{d} u,
\end{aligned}
$$

where the symbol \|\|$_{T}$ denotes the operator norm acting from the space $L_{1}[T, \infty)$ into itself. Now according to the fact that the set consisting of one element is weakly compact, by using Theorem 6 and the formula

$$
d(X)=\lim _{T \rightarrow \infty}\left\{\sup \left\{\int_{T}^{\infty}|x(t)| \mathrm{d} t: x \in X\right\}\right\} .
$$

and since $\lim _{T \rightarrow \infty} \varphi(T)=\infty$, we get

$$
d(H X) \leq\left(\frac{b\left\|K_{1}\right\|\left\|K_{2}\right\|}{M}\right) d(X) .
$$

According to Equation (3), combining (6) and (7), we get

$$
\mu(H X) \leq\left(\frac{b\left\|K_{1}\right\|\left\|K_{2}\right\|}{M}\right) \mu(X)
$$

Put $q=\left(b\left\|K_{1}\right\|\left\|K_{2}\right\|\right) / M$. Clearly, according to assumption iv) $q<1$. Consider the sequence of sets $\left\{B_{r}^{n}\right\}$, where $B_{r}^{1}=\operatorname{Conv}\left(G B_{r}\right), B_{r}^{2}=\operatorname{Conv}\left(G B_{r}^{1}\right)$ and so on. Obviously this sequence is decreasing i.e. $B_{r}^{n+1} \subset B_{r}^{n}$ for $n=1,2, \cdots$. Moreover, $B_{r}^{1} \subset B_{r}$. Apart from this, all sets belonging to this sequence are closed and convex, so weakly closed. On the other hand in view of inequality (8) we have

$$
\mu\left(B_{r}^{n}\right) \leq q^{n} \mu\left(B_{r}\right),
$$

which yields that $\lim _{n \rightarrow \infty} \mu\left(B_{r}^{n}\right)=0$.

Consequently, by axiom 5) of Definition 5 we infer that the set

$$
Y=\bigcap_{n=1}^{\infty} B_{r}^{n}
$$


is nonempty, closed, convex and weakly compact (in view of $\mu(Y)=0)$. Moreover, $G Y \subset Y$.

In the sequel we show that the set $G Y$ is relatively compact in the set $L_{1}$.

To do this let us take an arbitrary sequence $\left\{y_{n}\right\} \subset Y$ and fix arbitrarily a number $\varepsilon>0$. Since $Y$ is weakly compact, in view of Theorem 6 we deduce that there exists $T>0$ such that for any natural number $n$ the following inequality is satisfied

$$
\int_{T}^{\infty}\left|y_{n}(t)\right| \mathrm{d} t \leq \frac{\varepsilon}{4} .
$$

To apply the classical Schauder fixed point theorem, we need to prove that the set $H Y$ is relatively compact in $L_{1}$. For this aim let us consider the functions $f(t, x)$ on the set $[0, T]$ and the functions $k_{i}(t, s)$ on the set $[0, T] \times[0, T](i=1,2)$.

In view of Theorem 3 we can find a closed subset $D_{\varepsilon}$ of the interval $[0, T]$ such that meas. $\left(D_{\varepsilon}^{c}\right) \leq \varepsilon$ (where $\left.D_{\varepsilon}^{c}=[0, T] \backslash D_{\varepsilon}\right)$ and such that the functions $\left.f\right|_{D_{\varepsilon} \times R}$ and $\left.k_{i}\right|_{D_{\varepsilon} \times[0, T]}(i=1,2)$ are continuous. Hence we infer that $\left.k_{i}\right|_{D_{\varepsilon} \times[0, T]}(i=1,2)$ are uniformly continuous.

In what follows we show that $\left\{y_{n}\right\}$ is an equicontinuous on $D_{\varepsilon}$, for that let us take arbitrarily $t_{1}, t_{2} \in D_{\varepsilon}$. Without loss of generality we can assume that $t_{1}<t_{2}$. Then, keeping in mind our assumptions, for an arbitrary fixed $n \in N$ we obtain:

$$
\begin{aligned}
& \left|H y_{n}\left(t_{2}\right)-H y_{n}\left(t_{1}\right)\right| \\
& \leq \mid \int_{0}^{t_{2}} k_{1}\left(t_{2}, s\right) f\left(s, \int_{0}^{s} k_{2}(s, \theta) y_{n}(\varphi(\theta)) \mathrm{d} \theta\right) \mathrm{d} s \\
& \quad-\int_{0}^{t_{1}} k_{1}\left(t_{1}, s\right) f\left(s, \int_{0}^{s} k_{2}(s, \theta) y_{n}(\varphi(\theta)) \mathrm{d} \theta\right) \mathrm{d} s \mid \\
& \leq \omega^{T}\left(k_{1},\left|t_{2}-t_{1}\right|\right) \int_{0}^{T}\left\{a(s)+b \bar{k}_{2} \int_{0}^{s}\left|y_{n}(\varphi(\theta))\right| \mathrm{d} \theta\right\} \mathrm{d} s \\
& \quad+\bar{k}_{1} \int_{t_{1}}^{t_{1}}\left\{a(s)+b \bar{k}_{2} \int_{0}^{s}\left|y_{n}(\varphi(\theta))\right| \mathrm{d} \theta\right\} \mathrm{d} s
\end{aligned}
$$

where $\omega^{T}\left(k_{1}, \cdot\right)$ denotes the modulus of continuity of the function $k_{1}$ on the set $D_{\varepsilon} \times[0, T]$ and

$$
\begin{aligned}
& \bar{k}_{i}=\max \left\{\left|k_{i}(t, s)\right|:(t, s) \in D_{\varepsilon} \times[0, T], i=1,2\right\} . \\
& \left|H y_{n}\left(t_{2}\right)-H y_{n}\left(t_{1}\right)\right| \\
& \leq \omega^{T}\left(k_{1},\left|t_{2}-t_{1}\right|\right)\left\{\int_{0}^{T} a(s) \mathrm{d} s+b \bar{k}_{2} \int_{0}^{T} \int_{0}^{s}\left|y_{n}(\varphi(\theta))\right| \mathrm{d} \theta \mathrm{d} s\right\} \\
& \quad+\bar{k}_{1} \int_{t_{1}}^{t_{2}} a(s) \mathrm{d} s+b \bar{k}_{1} \bar{k}_{2} \int_{t_{1}}^{t_{2}} \int_{0}^{s}\left|y_{n}(\varphi(\theta))\right| \mathrm{d} \theta \mathrm{d} s
\end{aligned}
$$

By rearranging the order of double integrations, we get

$$
\begin{aligned}
& \left|H y_{n}\left(t_{2}\right)-H y_{n}\left(t_{1}\right)\right| \leq \omega^{T}\left(k_{1}, t_{2}-t_{1}\right) \\
& \cdot\left\{\int_{0}^{T} a(s) \mathrm{d} s+b \bar{k}_{2} \int_{0}^{T} \int_{\theta}^{T}\left|y_{n}(\varphi(\theta))\right| \mathrm{d} s \mathrm{~d} \theta\right\} \\
& +\bar{k}_{1} \int_{t_{1}}^{t_{2}} a(s) \mathrm{d} s+b \bar{k}_{1} \bar{k}_{2} \int_{0}^{t_{1}} \int_{t_{1}}^{t_{2}}\left|y_{n}(\varphi(\theta))\right| \mathrm{d} s \mathrm{~d} \theta \\
& +b_{1} \bar{k}_{1} \bar{k}_{2} \int_{t_{1}}^{t_{2}} \int_{\theta}^{t_{2}}\left|y_{n}(\varphi(\theta))\right| \mathrm{d} s \mathrm{~d} \theta \\
& \leq \omega^{T}\left(k^{1}, t_{2}-t^{1}\right)\left\{\int_{0}^{T} a(s) \mathrm{d} s+b \bar{k}_{2} T \int_{0}^{T}\left|y_{n}(\varphi(\theta))\right| \mathrm{d} \theta\right\} \\
& +\bar{k}_{1}^{t_{2}} \int_{t_{1}}^{t_{2}} a(s) \mathrm{d} s+b \bar{k}_{1} \bar{k}_{2}\left(t_{2}-t_{1}\right) \int_{0}^{t_{1}}\left|y_{n}(\varphi(\theta))\right| \mathrm{d} \theta \\
& +b \bar{k}_{1} \bar{k}_{2}\left(t_{2}-t_{1}\right) \int_{t_{1}}^{t_{2}}\left|y_{n}(\varphi(\theta))\right| \mathrm{d} \theta
\end{aligned}
$$

From the above estimate and the consideration of the fact that $Y \subset B_{r}$ we obtain

$$
\begin{aligned}
& \left|H y_{n}\left(t_{2}\right)-H y_{n}\left(t_{1}\right)\right| \leq \omega^{T}\left(k_{1}, t_{2}-t_{1}\right) \\
& \cdot\left\{\int_{0}^{\infty} a(s) \mathrm{d} s+b \bar{k}_{2} T \int_{0}^{\infty}\left|y_{n}(\varphi(\theta))\right| \mathrm{d} \theta\right\} \\
& +\bar{k}_{1}^{t_{2}} \int_{t_{1}} a(s) \mathrm{d} s+b \bar{k}_{1} \bar{k}_{2}\left(t_{2}-t_{1}\right) \int_{0}^{t_{1}}\left|y_{n}(\varphi(\theta))\right| \mathrm{d} \theta \\
& +b \bar{k}_{1} \bar{k}_{2}\left(t_{2}-t_{1}\right) \int_{t_{1}}^{t_{2}}\left|y_{n}(\varphi(\theta))\right| \mathrm{d} \theta \\
& \leq \omega^{T}\left(k_{1}, t_{2}-t_{1}\right)\left\{a+\frac{b T \bar{k}_{2}}{M} r\right\}+\bar{k}_{1} \int_{t_{1}}^{t_{2}} a(s) \mathrm{d} s \\
& +\frac{b \bar{k}_{1} \bar{k}_{2}\left(t_{2}-t_{1}\right)}{M} r .
\end{aligned}
$$

Now, utilizing the fact that the sequence $\left\{y_{n}\right\}$ is weakly compact and taking into account Theorem 6 we can show that the number

$$
\int_{t_{1}}^{t_{2}} a(s) \mathrm{d} s
$$

is arbitrarily small provided the number $\left(t_{2}-t_{1}\right)$ is taken to be sufficiently small (it is a consequence of the fact that a one element set is weakly compact in $L_{1}$ ).

Furthermore, 


$$
\begin{aligned}
& \left\|\left(H y_{n}\right)(t)\right\| \\
& =\left|\int_{0}^{t} k_{1}(t, s) f\left(s, \int_{0}^{s} k_{2}(s, \theta) y_{n}(\varphi(\theta)) \mathrm{d} \theta\right) \mathrm{d} s\right| \\
& \leq \int_{0}^{t}\left|k_{1}(t, s)\right|\left\{a(s)+b \int_{0}^{s}\left|k_{2}(s, \theta)\right|\left|y_{n}(\varphi(\theta))\right| \mathrm{d} \theta\right\} \mathrm{d} s \\
& \leq \bar{k}_{1} \int_{0}^{t} a(s) \mathrm{d} s+b \bar{k}_{1} \bar{k}_{2} \int_{0}^{t} \int_{\theta}^{t}\left|y_{n}(\varphi(\theta))\right| \mathrm{d} s \mathrm{~d} \theta \\
& \leq \bar{k}_{1} \int_{0}^{t} a(s) \mathrm{d} s+b \bar{k}_{1} \bar{k}_{2} \int_{0}^{t}\left|y_{n}(\varphi(\theta))\right| \int_{\theta}^{t} \mathrm{~d} s \mathrm{~d} \theta \\
& \leq \bar{k}_{1} \int_{0}^{t} a(s) \mathrm{d} s+b \bar{k}_{1} \bar{k}_{2} \int_{0}^{t}\left|y_{n}(\varphi(\theta))\right|(t-\theta) \mathrm{d} \theta \\
& \leq \bar{k}_{1} \int_{0}^{t} a(s) \mathrm{d} s+b \overline{k_{1}} \bar{k}_{2} T \int_{0}^{t}\left|y_{n}(\varphi(\theta))\right| \mathrm{d} \theta \\
& \leq \bar{k}_{1} \int_{0}^{t} a(s) \mathrm{d} s+b \bar{k}_{1} \bar{k}_{2} T \int_{0}^{t}\left|y_{n}(\varphi(\theta))\right| \frac{\varphi^{\prime}(\theta)}{M} \mathrm{~d} \theta \\
& \leq \bar{k}_{1} \int_{0}^{t} a(s) \mathrm{d} s+\frac{b \bar{k}_{1} \bar{k}_{2} T}{M} \int_{0}^{\varphi(t)}\left|y_{n}(u)\right| \mathrm{d} u
\end{aligned}
$$

Hence

$$
\left\|\left(H y_{n}\right)(t)\right\| \leq \bar{k}_{1}\|a\|+b \bar{k}_{1} \bar{k}_{2} T M^{-1} r .
$$

Hence consequently the sequence $\left\{H y_{n}\right\}$ is a sequence of uniformly bounded and equicontinuous functions on $D_{\varepsilon}$. Hence, in view of Ascoli-Arzela theorem we deduce that the sequence $\left\{H y_{n}\right\}$ is relatively compact subset in the space $C\left(D_{\varepsilon}\right)$.

Further observe that the above reasoning does not depend on the choice of $\varepsilon$. Thus we can construct a sequence $\left\{D_{p}\right\}$ of closed subsets of the interval $[0, T]$ such that meas. $\left(D_{p}^{c}\right) \rightarrow 0$ as $p \rightarrow \infty$ and such that the sequence $\left\{H y_{n}\right\}$ is relatively compact in every space $C\left(D_{p}\right)$. Passing to subsequences if necessary we can assume that $\left\{\mathrm{Hy}_{n}\right\}$ is a Cauchy sequence in each space $C\left(D_{p}\right)$, for $p=1,2, \cdots$.

In what follows, utilizing the fact that the set $H Y$ is weakly compact, let us choose a number $\delta>0$ such that for each closed subset $D_{\delta}$ of the interval $[0, T]$ such that meas. $\left(D_{\delta}^{c}\right) \leq \delta$ we have

$$
\int_{D_{\delta}^{c}}|(H y)(t)| \mathrm{d} t \leq \frac{\varepsilon}{4}
$$

for any $y \in Y$.

Keeping in mind the fact that the sequence $\left\{H y_{n}\right\}$ is a Cauchy sequence in each space $C\left(D_{p}\right)$ we can choose a natural number $p_{0}$ such that meas. $\left(D_{p_{0}}^{c}\right) \leq \delta$ and for arbitrary natural numbers $n, m \geq p_{0}$ the following inequality holds

$$
\left|\left(H y_{n}\right)(t)-\left(H y_{m}\right)(t)\right| \leq \frac{\varepsilon}{4 \text { meas. }\left(D_{p_{0}}\right)}
$$

for any $t \in D_{p_{0}}$. Obviously without loss of generality we can assume that meas. $\left(D_{p_{0}}\right)>0$.

Now, using the above facts and (10) we obtain

$$
\begin{aligned}
& \int_{0}^{T}\left|\left(H y_{n}\right)(t)-\left(H y_{m}\right)(t)\right| \mathrm{d} t \\
& =\int_{D_{p_{0}}}\left|\left(H y_{m}\right)(t)-\left(H y_{m}\right)(t)\right| \mathrm{d} t \\
& +\int_{D_{p_{0}}^{c}}\left|\left(H y_{m}\right)(t)-\left(H y_{m}\right)(t)\right| \mathrm{d} t \\
& \leq \frac{\varepsilon \text { meas. }\left(D_{p^{0}}\right)}{4 \text { meas. }\left(D_{p^{0}}\right)} \\
& +\int_{D_{p_{0}}^{c}}\left\{\left|\left(H y_{m}\right)(t)\right|+\left|\left(H y_{m}\right)(t)\right|\right\} \mathrm{d} t=\frac{3 \varepsilon}{4} .
\end{aligned}
$$

Finally, from (10) and (11) we get

$$
\left\|H y_{m}-H y_{n}\right\|=\int_{0}^{\infty}\left|\left(H y_{n}\right)(t)-\left(H y_{m}\right)(t)\right| \mathrm{d} t \leq \varepsilon,
$$

which means that $\left\{H y_{n}\right\}$ is a Cauchy sequence in the space $L_{1}=L_{1}\left(R_{+}\right)$. Hence we conclude that the set $H Y$ is relativelycompact in this space.

In the last step of the proof let us consider the set $Y_{0}=\operatorname{Conv}(H Y)$. In view of the Mazur theorem we infer that the set $Y_{0}$ is compact in the space $L_{1}$. Moreover, we have that the operator $H$ transforms continuously the set $Y_{0}$ into itself. Thus the classical Schauder fixed point principle gives that $H$ has at least one fixed point. This proves that there exists at least one $x \in L_{1}$ that solves Equation (4).

\section{Nonlinear Equation of Convolution Type}

Assume that $k: R_{+} \rightarrow R$ is an integrable function. For an arbitrary function $x \in L_{1}$ set

$$
(K x)(t)=\int_{0}^{t} k(t-s) x(s) \mathrm{d} s, t \in R_{+} .
$$

This operator $K$ is a linear integral operator of convolution type and maps $L_{1}$ into itself continuously. Now, consider the following condition

$$
(v) k: R_{+} \rightarrow R \text { and } k \in L_{1} \text {. }
$$

Then we have the following Corollary

Corollary 11. Let the hypotheses i)-v) are satisfied. Then a nonlinear equation of convolution type

$$
\begin{aligned}
& x(t)=\int_{0}^{t} k_{1}(t, s) f\left(s, \int_{0}^{s} k_{2}(s-\theta) x(\varphi(\theta)) \mathrm{d} \theta\right) \mathrm{d} s, \\
& t \in R_{+}
\end{aligned}
$$


has at least one integrable solution $x \in L_{1}$.

In the next subsection, we prove an existence theorem for integral equation of fractional order as a special form of Equation (12).

\section{Initial Value Problems of Fractional Order}

As a special case of Equation (14), we consider

$$
x(t)=\int_{0}^{t} k_{1}(t, s) f\left(s, \int_{0}^{s} \frac{(s-\theta)^{-\beta}}{\Gamma(1-\beta)} x(\theta) \mathrm{d} \theta\right) \mathrm{d} s, t \in R_{+}
$$

where

$$
k_{2}(s-\theta)=\frac{(s-\theta)^{-\beta}}{\Gamma(1-\beta)}, \beta \in(0,1]
$$

and $\varphi(\theta)=\theta$. Equation (13) is an integral equation of fractional order that can be written in the form

$$
x(t)=\int_{0}^{t} k_{1}(t, s) f\left(s, I^{1-\beta} x(s) \mathrm{d} s\right), t \in R_{+}
$$

Obviously, Equation (14) has at least one integrable solution $x \in L_{1}$.

Definition 12. By a solution of the initial value problem (1) we mean an absolutely continuous function $x$ satisfies the initial value problem (1).

Theorem 13. Let $b\left\|K_{2}\right\| / \Gamma(2-\beta)<1$ and $\beta \in(0,1]$. If assumptions i)-iii) and v) are satisfied, then the initial value problem (1) has at least one solution $y \in L_{1}$.

Proof. Let $x$ be a solution of the integral Equation (14). Putting

$$
y(t)=\int_{0}^{t} x(\tau) \mathrm{d} \tau
$$

Since $x$ is integrable, then

$$
D y(t)=D \int_{0}^{t} x(\tau) \mathrm{d} \tau \text { a.e. }
$$

where $D=\frac{\mathrm{d}}{\mathrm{d} t}$. Moreover, the integral $\int_{0}^{t} x(\tau) \mathrm{d} \tau$ of integrable function $x$ is absolutely continuous then

$$
D y(t)=D I^{1} x(t)
$$

Then we have,

$$
D y(t)=x(t) \text { a.e. }
$$

Furthermore, we obtain

$$
\begin{aligned}
& D y(t)=x(t) \\
& I^{1-\beta} D y(t)=I^{1-\beta} x(t) \\
& D^{\beta} y(t)=I^{1-\beta} x(t)
\end{aligned}
$$

Consequently, Equation (14) gives

$$
\frac{\mathrm{d}}{\mathrm{d} t} y(t)=D y(t)=\int_{0}^{t} k_{1}(t, s) f\left(s, D^{\beta} y(s) \mathrm{d} s\right),
$$

Since $x$ is integrable and absolutely continuous, then

$$
\begin{aligned}
& D y(\tau)=x(\tau) \\
& I^{1} D y(\tau)=I^{1} x(\tau) \\
& \int_{0}^{t} \frac{\mathrm{d}}{\mathrm{d} t} y(\tau) \mathrm{d} \tau=\int_{0}^{t} x(\tau) \mathrm{d} \tau \\
& y(t)-y_{0}=\int_{0}^{t} x(\tau) \mathrm{d} \tau
\end{aligned}
$$

Clearly, $y(0)=y_{0}$. Hence we deduce that $y$ is an absolutely continuous function satisfies the initial value problem (1). Hence the proof is complete.

\section{Conclusion}

The existence theorem of functional integrable equation in the space of Lebesgue integrable functions on unbounded interval $L_{1}[0, \infty)$ is presented and proved. As an application of this theorem, we investigated the existence of solution of the suggested initial value problems of fractional order.

\section{REFERENCES}

[1] J. Banas and Z. Knap, "Integrable Solutions of a Functional-Integral Equation," Revista Matemática de la Universidad Complutense de Madrid, Vol. 2, No. 1, 1989, pp. 31-38.

[2] J. Banas and A. Chlebowicz, "On Existence of Integrable Solutions of a Functional Integral Equation under Carathéodory Conditions,” Nonlinear Analysis, Vol. 70, No. 9, 2009, pp. 3172-3179.

[3] G. Emmanuele, "Integrable Solutions of a Functional Integral Equation," Journal of Integral Equations and Applications, Vol. 4, No. 1, 1992, pp. 89-94. doi:10.1216/jiea/1181075668

[4] P. P. Zabrejko, A. I. Koshelev, M. A. Krasnosel'skii, S. G. Mikhlin, L. S. Rakovshchik and V. J. Stecenko, "Integral Equations,” Noordhoff, Leyden, 1975.

[5] A. M. A. El-Sayed, "Nonlinear Functional Differential Equations of Arbitrary Orders,” Nonlinear Analysis, Vol. 33, No. 2, 1998, pp. 181-186. doi:10.1016/S0362-546X(97)00525-7

[6] A. M. A. El-Sayed, N. Sherif and I. A. Ibrahim, "On a Mixed Type Integral Equation and Fractional Order Functional Differential Equations," Commentationes Mathematicae. Prace Matematyczne, Vol. 45, No. 2, 2005, pp. 237-247.

[7] I. A. Ibrahim, T. S. Amer and Y. M. Abo Essa, "Integrable Solutions of Initial Value Problems of Fractional Order,” Far East Journal of Mathematical Sciences, Vol. 62, No. 1, 2012, pp. 97-123.

[8] J. Appel, "Implicit Functions, Nonlinear Integral Equa- 
tions and the Measure of Noncompactness of the Superposition Operator," Journal of Mathematical Analysis and Applications, Vol. 83, No. 1, 1981, pp. 251-263. doi:10.1016/0022-247X(81)90261-4

[9] M. A. Krasnosel'skii, P. P. Zabrejko, J. I. Pustyl'nik and P. J. Sobolevskii, "Integral Operators in Spaces of Summable Functions,” Noordhoff, Leyden, 1976.

[10] R. Pluciennik, "On Some Properties of the Superposition Operator in Generalized Orlicz Spaces of Vector-Valued Functions," Commentationes Mathematicae. Prace Matematyczne, Vol. 25, No. 2, 1985, pp. 321-337.

[11] K. Carathéodory, "Vorlesungen Über Reele Funktionen," De Gruyter, Leipzig, 1918.

[12] J. Appel and P. P. Zabrejko, "Nonlinear Superposition Operators," In: Cambridge Tracts in Mathematics, Vol. 95, Cambridge University Press, Cambridge, 1990.

[13] G. Scorza Dragoni, "Un Teorema Sulle Funzioni Continue Rispetto ad une e Misarubili Rispetto ad Un’altra Variable," Rendiconti del Seminario Matematico della Università di Padova, 1948, pp. 102-106.

[14] N. Dunford and J. T. Schwartz, "Linear Operators," Int. Publ., Leyden, 1963.

[15] J. Banas and W. G. El-Sayed, "Measures of Noncompactness and Solvability of an Integral Equation in the
Class of Functions of Locally Bounded Variaton,” Journal of Mathematical Analysis and Applications, Vol. 167, No. 1, 1992, pp. 133-151. doi:10.1016/0022-247X(92)90241-5

[16] J. Banas and J. Rivero, "On Measures of Weak Noncompactness,” Annali di Matematica Pura ed Applicata, Vol. 151, No. 1, 1988, pp. 213-224. doi:10.1007/BF01762795

[17] J. Banas and Z. Knap, "Measures of Weak Noncompactness and Nonlinear Integral Equations of Convolution Type,” Journal of Mathematical Analysis and Applications, Vol. 146, No. 2, 1990, pp. 353-362. doi:10.1016/0022-247X(90)90307-2

[18] J. Dieudonné, “Sur les Espaces de Köthe,” Journal d’Analyse Mathématique, Vol. 1, No. 1, 1951, pp. 81-115. doi:10.1007/BF02790084

[19] A. M. A. El-Sayed, W. G. El-Sayed and O. L. Moustafa, “On Some Fractional Functional Equations," Pure Mathematics and Applications, Vol. 6, No. 4, 1995, pp. 321332.

[20] S. G. Samko, A. A. Kilbas and O. I. Marichev, "Fractional Integrals and Derivatives Theory and Applications," Gordon and Breach Science Publishers, Amsterdam, 1993. 